\title{
PATHWEIGH, pragmatic weight management in adult patients in primary care in Colorado, USA: study protocol for a stepped wedge cluster randomized trial
}

Krithika Suresh ${ }^{1 *}$ D, Jodi Summers Holtrop ${ }^{2}$, L. Miriam Dickinson², Emileigh Willems ${ }^{1}$, Peter C. Smith², R. Mark Gritz ${ }^{2}$ and Leigh Perreault ${ }^{1,2}$

\begin{abstract}
Background: Despite the overwhelming prevalence and health implications of obesity, it is rarely adequately addressed in a health care setting. PATHWEIGH is a pragmatic approach to weight management that uses tools built into the electronic medical record to overcome barriers and guide care. Implementation strategies are employed to facilitate adoption and use of the PATHWEIGH tools and processes. The current study will compare the effectiveness of PATHWEIGH versus standard of care (SOC) on patient weight loss in primary care and explore factors for its successful implementation.
\end{abstract}

Methods: A stepped wedge cluster randomized trial design will be used within an effectiveness-implementation hybrid study. Adult patient weight loss and weight loss maintenance will be compared in PATHWEIGH versus SOC in 57 family and internal medicine clinics in a large health system in Colorado, USA. Effectiveness will be evaluated using generalized linear mixed models to determine statistical differences in weight loss and weight loss maintenance at 6, 12, and 18 months. Patient-, provider-, and clinic-level predictors will be identified using mediator and moderator analyses. Conceptually guided by the Practical, Robust, Implementation and Sustainability Model (PRISM), a mixed methods approach including quantitative (practice surveys, use tracking) and qualitative (interviews, observations) data collection will be used to determine factors impeding and facilitating adoption, implementation, and maintenance of PATHWEIGH and evaluate specified implementation strategies. A cost analysis of the practice and system costs and resources required by PATHWEIGH relative to the reimbursement collected will be performed.

Discussion: The effectiveness and implementation of PATHWEIGH, and their interrelatedness, for patient weight loss are collectively the focus of the current trial. Findings from this study are expected to serve as a blueprint for available and effective weight management in primary care medical practice.

Trial registration: ClinicalTrials.gov NCT04678752. Registered on December 21, 2020.

Keywords: Cluster randomized trial, Mixed methods, Pragmatic trial, PRISM, RE-AIM, Stepped wedge, Weight loss

\footnotetext{
* Correspondence: krithika.suresh@cuanschutz.edu

${ }^{1}$ Colorado School of Public Health, 13001 East 17th Place, Aurora, CO 80045,

USA

Full list of author information is available at the end of the article
}

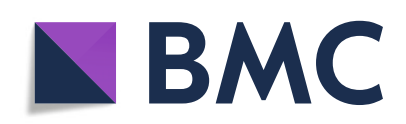

(- The Author(s). 2022 Open Access This article is licensed under a Creative Commons Attribution 4.0 International License, which permits use, sharing, adaptation, distribution and reproduction in any medium or format, as long as you give appropriate credit to the original author(s) and the source, provide a link to the Creative Commons licence, and indicate if changes were made. The images or other third party material in this article are included in the article's Creative Commons licence, unless indicated otherwise in a credit line to the material. If material is not included in the article's Creative Commons licence and your intended use is not permitted by statutory regulation or exceeds the permitted use, you will need to obtain permission directly from the copyright holder. To view a copy of this licence, visit http://creativecommons.org/licenses/by/4.0/. The Creative Commons Public Domain Dedication waiver (http://creativecommons.org/publicdomain/zero/1.0/) applies to the data made available in this article, unless otherwise stated in a credit line to the data. 


\section{Background}

In the USA, obesity prevalence continues to increase. In 2019, the Centers for Disease Control and Prevention (CDC) estimated that $70 \%$ of US adults were considered overweight and $42 \%$ had obesity [1]. Obesity is a wellestablished risk factor for numerous diseases, including diabetes, cardiovascular disease, cancer, immobility, mental health issues, as well as all-cause mortality [2]. Obesity is also a strong and independent risk factor for poor outcomes due to COVID-19 [3]. In 2018, direct and indirect costs associated with obesity in the USA reached \$1.7 T [4]. Importantly, obesity is being increasingly recognized not only as a risk factor for disease, but a disease unto itself [2]. Despite this, obesity is rarely addressed in a healthcare setting. Only $\sim 50 \%$ of people with a BMI of $50 \mathrm{~kg} / \mathrm{m}^{2}$ (considered morbid or extreme obesity) have a documented diagnosis of obesity [5] and $<1 \%$ of people with any degree of overweight or obesity are offered anything other than lifestyle advice [6].

Reasons behind the lack of weight management prioritization in clinical settings are extensive and complex. Health care providers cite lack of time, education, and resources, as well as competing issues, as the leading reasons why obesity is not prioritized [7-9], but poor reimbursement and lack of effective tools are also widely cited $[7,8]$. Further, lack of data on long-term effectiveness of weight loss treatments leads to uncertainty and learned helplessness among both health care providers and patients. Eighty-two percent of people with obesity believe they are responsible for their weight; a number highly corroborated by health care providers, enhancing shame and hesitancy to seek help [7]. While these are only a few of the reasons why weight is rarely addressed, increasing evidence suggests barriers can be overcome by intentionally employing long-term evidence-based strategies. Importantly, such strategies must be employed where patients access care, most notably in primary care.

Primary care is undergoing rapid transformation with a shift toward population-based approaches that prioritize preventive care. Initiatives such as pay for performance, the Patient-Centered Medical Home [10], and efforts associated with the Affordable Care Act [11] have brought a host of structures, tools, and processes not previously available, creating new opportunities to engage patients in efficacious programs. Many practices now have electronic medical records (EMRs), patient registries, and a team approach to care that may include expanded care visits with care managers, dietitians, and social workers or psychologists [12, 13]. Increasingly, value-based payment models incentivize practices to identify patients at high risk for complications of chronic conditions and to mitigate these risks. Many of these chronic conditions can be improved or alleviated by reducing excess weight.
Importantly, patient demand is high for weight management in primary care. A study by Sherson et al. [14] reported that the majority of patients want to discuss weight loss with their physicians. Specifically, patients value physician direction with their diet, physical activity, and goal setting [15]. In addition, recent years have ushered in numerous and diverse options for weight management that extend significantly beyond lifestyle advice. Medications for weight loss are being more extensively studied and demonstrating improved outcomes [16-19]. Bariatric surgery provides a possible option to address weight and reverse potentially life-threatening conditions such as heart disease and diabetes in both adolescents and adults [20-23]. Intensive behavioral therapy (IBT) for obesity is now a covered benefit under Medicare [24]. Together, primary care practice transformation, reimbursement for obesity care, and better therapies for weight management suggest new and pragmatic approaches can emerge.

In the Fall of 2020, the National Institutes of Health (NIH) National Institute of Diabetes and Digestive and Kidney Diseases (NIDDK) funded "PATHWEIGH: pragmatic weight management in primary care" (1R18DK127003). The aim of PATHWEIGH is to create the blueprint for pragmatic, scalable, and sustainable weight management in primary care using existing resources. To achieve this aim, this study will deploy an effectiveness-implementation hybrid type 1 [25] stepped wedge cluster randomized trial design [26] in 57 healthsystem owned family medicine and general internal medicine clinics that span diverse settings, patient populations, and community contexts in Colorado.

\section{Aims and hypotheses}

The specific aims of the current research are to:

1. Compare the effectiveness of PATHWEIGH versus standard of care (SOC) on weight loss and weight loss maintenance.

2. Identify patient, provider, and clinic-level predictors that are associated with weight loss and weight loss maintenance.

3. Describe factors associated with practice adoption, implementation, and maintenance of PATHWEIGH.

We hypothesize that PATHWEIGH will lead to greater weight loss at 6 months (primary) and greater weight loss maintenance at 12 and 18 months (secondary) compared to SOC [Hypothesis I]. Key patient, clinician, and clinic characteristics will mediate and moderate patient weight loss and weight loss maintenance [Hypothesis II]. Effective implementation will be 
vital to anticipated implementation and clinical outcomes [Hypothesis III].

\section{Methods}

The study methods are described in compliance with the SPIRIT statement [27] and the Consolidated Standards of Reporting Trials (CONSORT) extension for stepped wedge cluster randomized trials [28] to ensure appropriate reporting of the study protocol and completed trial. The SPIRIT checklist is provided in the Additional file.

\section{Study setting}

This study will be conducted in 57 family medicine and general internal medicine clinics within a large, multi-specialty health care system in Colorado (Fig. 1 ). One primary care clinic was excluded as it was a pilot test site [29]. These practices are comprised of approximately 400 clinician full-time equivalents (physician, nurse practitioner, physician assistant) caring for nearly 500,000 patients in diverse geographic and socioeconomic contexts. All practices have the following resources for the implementation of obesity management: access and full use of the Epic EMR, practice quality improvement facilitators, standardized workflows for medical assistants, funds for site innovation, inter-connectedness to the system at large, and regular training opportunities. Primary care leadership has unanimously offered their support for this project.

\section{Design and randomization}

We will use a type 1 hybrid effectivenessimplementation stepped wedge cluster randomized design to assess both patient-level clinical and practicelevel implementation outcomes [25, 26]. The intervention will be implemented sequentially in the 57 participating clinics in three steps over a 4-year period (Fig. 2). All sites will start in the control condition of SOC for weight management. Every year, one sequence of clinics will transition to the intervention condition after which the use of PATHWEIGH will be encouraged for all weight-prioritized visits and corresponding PATHWEIGH implementation strategies will be provided. Clinics were randomly assigned by the study statistician to each sequence using covariate constrained randomization [30] to achieve balance on clinic type (academic, affiliate, non-academic), clinic location (urban/suburban vs. rural), provider type (family medicine (FM), general internal medicine (GIM), or both), annual number of patient visits, and patient insurance status (percentage Medicaid) (Table 1). A stepped wedge extension for this procedure was used to account for the proportion of the study period that each of the sites spend in the control and intervention periods [30]. For additional details about the randomization procedure see Additional file.

\section{Participant eligibility criteria}

Patients eligible for this analysis must (1) be aged 18 years or older, (2) be overweight (BMI $\geq 25 \mathrm{~kg} / \mathrm{m}^{2}$ ) at

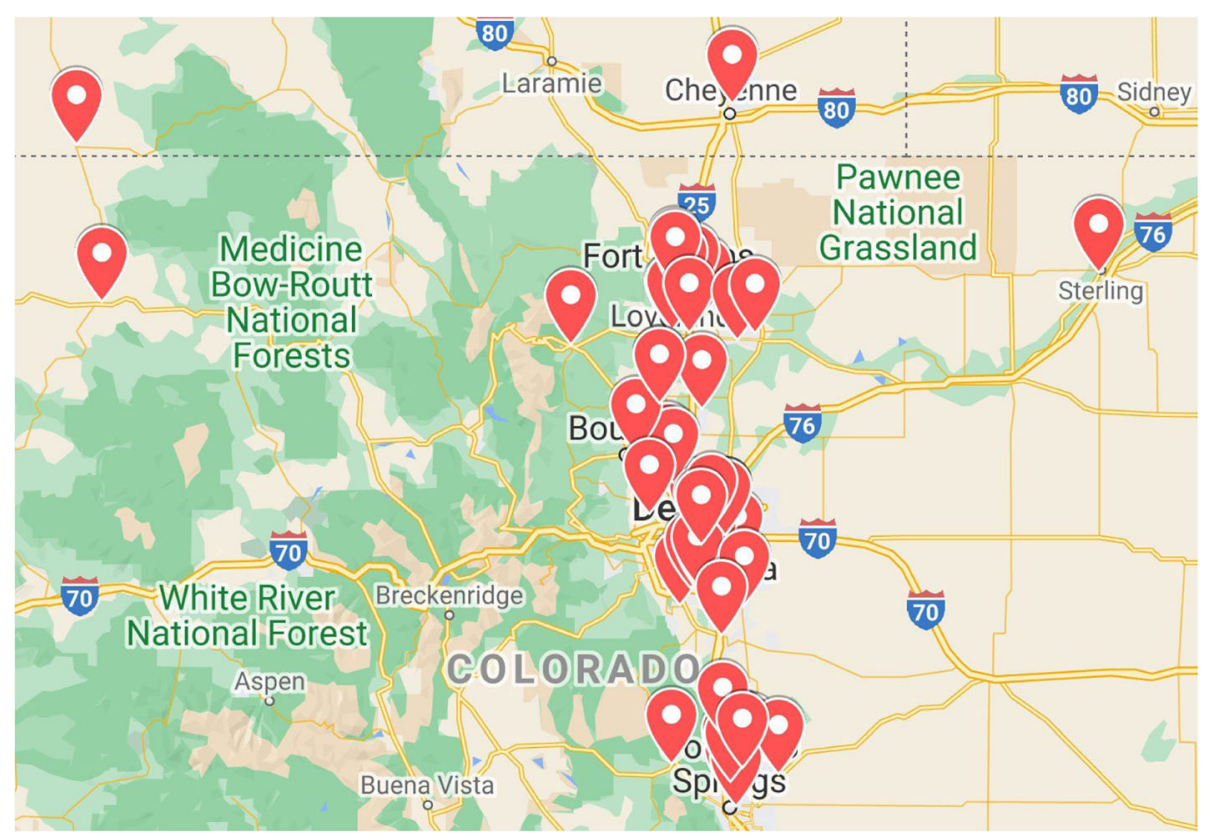

Fig. 1 Location of participating Colorado and Wyoming clinics. Image created using Google Map data 2022 


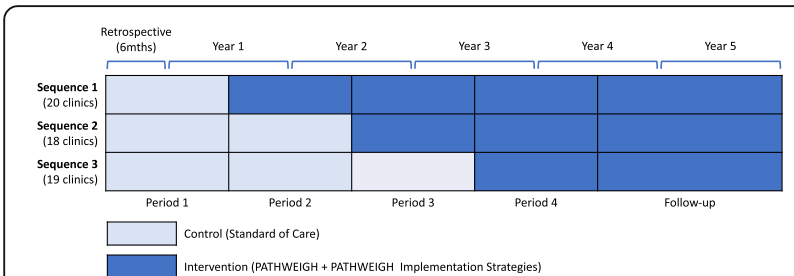

Fig. 2 Study diagram for the PATHWEIGH study, a stepped-wedge cluster hybrid type 1 randomized controlled trial design

initial visit, and (3) have had at least one weightprioritized visit in a participating clinic. Exclusion criteria in the original protocol include limited cognitive ability, have a non-home residence, or have less than 1year life expectancy. Ultimately, these were not applied due to the inability to obtain the necessary fields in the data extraction; however, the number of individuals that would be excluded based on these criteria is expected to be minimal and balanced across the study arms. Weightprioritized visits are defined as the chief complaint or reason for the visit being at least one of the following: (1) "overweight," "obesity," or "weight," (2) ICD-10-CM codes for billing E66-E.66.9, Z68.25-45, (3) use of the "obesity brief HPI" Epic flowsheet, or (4) the PATHWEIGH patient questionnaire.

\section{Data collection and management}

De-identified data will be extracted from Epic (the electronic medical record) from March 17, 2020, to March 16, 2021, then every 6 months from March 17, 2021, until September 17, 2025. The study timeline is depicted in a SPIRIT diagram in Fig. 3. Data extraction will be performed by Health Data Compass, the Colorado
Center for Personalized Medicine's cloud-based enterprise data warehouse and management platform. Health Data Compass extracts de-identified data using a proprietary process, wherein all data are checked for process compliance and missingness prior to release to the study team. As these data are de-identified, informed consent was not utilized. However, a written informed consent process was utilized for specified, individually contacted patients and practice members for the purposes of the implementation evaluation.

Retrospective data will be collected on all patient encounters at the 57 primary care clinics at the specified times described above. Specifically, how many patient visits occurred at each clinic, how many discrete patients were seen at each clinic, how many patients were $\geq 18$ years old with a BMI $\geq 25 \mathrm{~kg} / \mathrm{m}^{2}$, and how many patients who were $\geq 18$ years old with a BMI $\geq 25 \mathrm{~kg} / \mathrm{m}^{2}$ had a weight-prioritized visit (either in person or by telehealth). These data will be illustrated in an Expanded CONSORT diagram [31]. Data will be stored on a secure data server with access provided to the study statisticians. The statisticians will not be blinded to the assignment of the interventions.

\section{Sample size}

A conservative sample size estimate is 6840 total patients (120 patients per clinic from 57 clinics) that assume a minimum of 30 eligible patients per clinic per year based on the smallest clinic. We present the power calculations assuming an intraclass correlation coefficient (ICC) between 0.02 and 0.05 . With this design, we will have $80 \%$ power (alpha $=0.05$, two-sided) to detect a standardized effect size of $0.118 \quad(\mathrm{ICC}=0.02$ ) to 0.121 (ICC $=0.05)$. For our primary effectiveness outcomes, we

Table 1 Characteristics of participating practices and patients by randomization sequence

\begin{tabular}{|c|c|c|c|}
\hline Site characteristic & $\begin{array}{l}\text { Sequence } 1 \\
\text { (20 clinics) }\end{array}$ & $\begin{array}{l}\text { Sequence } 2 \\
\text { (18 clinics) }\end{array}$ & $\begin{array}{l}\text { Sequence } 3 \\
\text { (19 clinics) }\end{array}$ \\
\hline \multicolumn{4}{|l|}{ Type, $n$} \\
\hline Academic & 1 & 1 & 2 \\
\hline Affiliate & 4 & 2 & 2 \\
\hline Non-academic & 15 & 15 & 15 \\
\hline \multicolumn{4}{|l|}{ Location, $n$} \\
\hline Urban & 17 & 16 & 16 \\
\hline Rural & 3 & 2 & 3 \\
\hline \multicolumn{4}{|l|}{ Specialty, $n$} \\
\hline Family Medicine only (FM) & 10 & 9 & 11 \\
\hline General Internal Medicine only (GIM) & 6 & 6 & 4 \\
\hline Mixed FM/GIM & 4 & 3 & 4 \\
\hline Number of patients visits in 2019 , median (IQR) & $10,589(6059-22,233)$ & $13,579(7666-20,035)$ & $9815(3552-29,910)$ \\
\hline Percentage Medicaid, median (IQR) & $3.5(2.0-5.0)$ & $5.0(3.0-7.0)$ & $2.5(1.8-7.0)$ \\
\hline
\end{tabular}




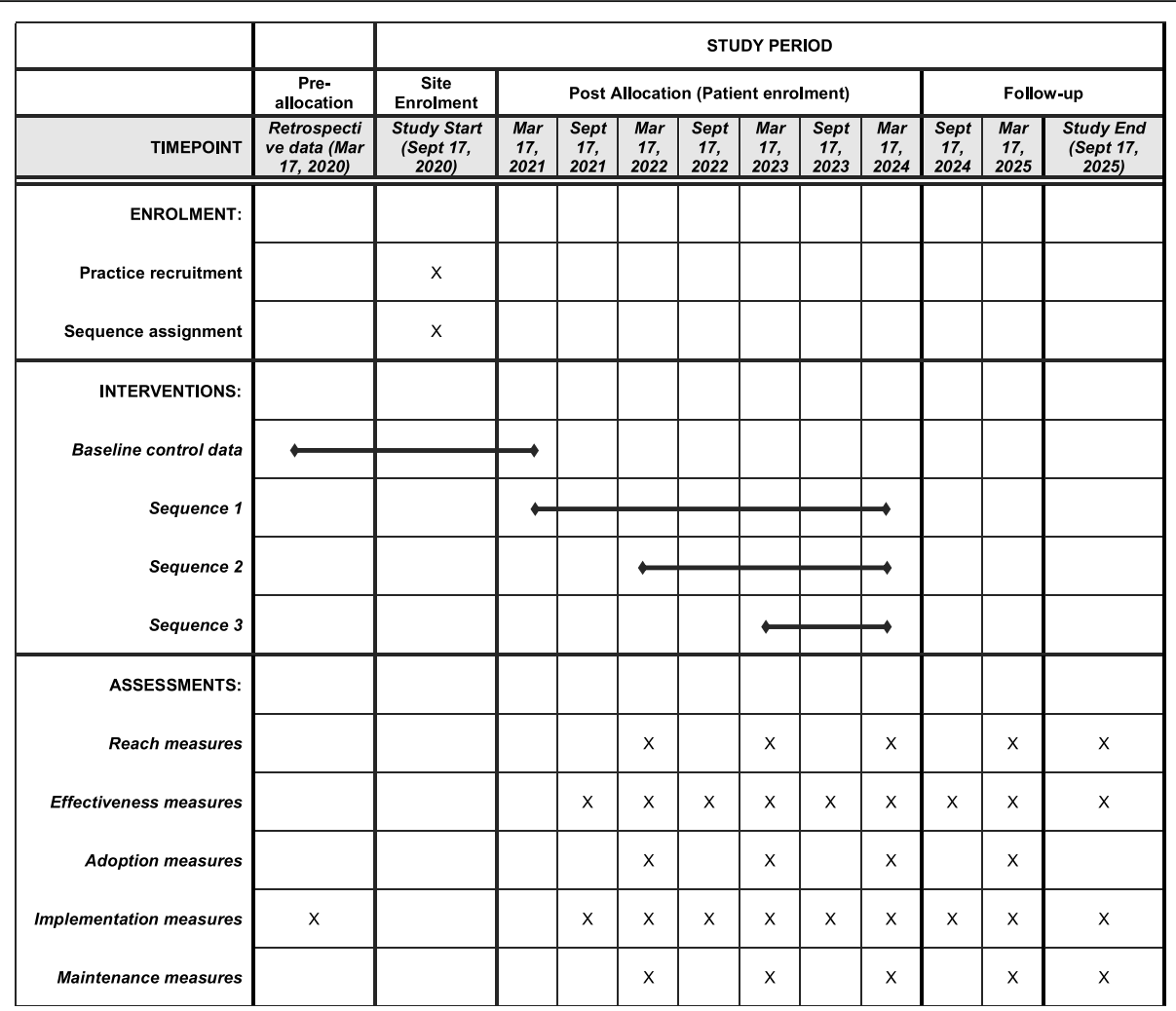

Fig. 3 SPIRIT diagram

estimate a standard deviation (SD) of 10.1 percentage points for percentage change in weight using our pilot study, and a similar SD estimate of 10.7 for absolute $(\mathrm{kg})$ change in weight. Thus, with $80 \%$ power we can detect a mean difference in weight change of $1.19(\mathrm{ICC}=0.02)$ to 1.22 (ICC $=0.05)$ percentage points between the control and intervention conditions, and a mean difference in absolute weight change of $1.26 \mathrm{~kg}(\mathrm{ICC}=0.02)$ to $1.29 \mathrm{~kg}$ $(\mathrm{ICC}=0.05)$.

\section{Interventions}

\section{Current standard of care (SOC)}

SOC will be the control condition that patients will receive during the control phases of the design. Clinics in the SOC condition will conduct visits in which weight may be discussed (defined as "weight-prioritized" for purposes of data extraction) without access to either the PATHWEIGH tools in Epic or the PATHWEIGH implementation strategies. Current SOC for obesity in the primary care clinics is called "obesity - brief history of present illness (HPI)," and the option to select "Obesity brief HPI" is currently available to all Epic users at all practices. For an example of the Obesity brief HPI questionnaires, see Additional File Fig. S1. For a weight prioritized visit, this entails a brief historical intake of a patient's weight and is intended to initiate a discussion regarding potential strategies for weight loss. Because SOC can be highly variable, we will conduct fidelity checks by performing sensitivity analyses for the use of the "Obesity brief HPI" in the SOC clinics at our every 6-month data extractions. A change in the use of the "Obesity brief HPI" by more than $30 \%$ between 6-month intervals will be investigated. There are currently no systematic weight management interventions at the clinics.

\section{PATHWEIGH: Clinical workflow and management}

PATHWEIGH is a workflow optimization and management system for weight management built into Epic (Fig. 4). The PATHWEIGH tools in Epic are designed to address numerous barriers to prioritizing weight management in primary care [8]. Once practices transition to receive the intervention, PATHWEIGH will be "turned on" within Epic and the PATHWEIGH implementation strategies will be provided. The key components of PATHWEIGH are summarized in Table 2 and include (1) encouraging patients to schedule a weightprioritized visit if they would like medical assistance with weight management, (2) improving workflow efficiency around weight-prioritized visits, and (3) clinical decision support during weight-prioritized visits. 


\begin{tabular}{l|l|l|l|}
\hline $\begin{array}{l}\text { Step 1. Ask patients if } \\
\text { they would like } \\
\text { medical assistance } \\
\text { with weight } \\
\text { management. If so, } \\
\text { they should schedule } \\
\text { a "weight-prioritized } \\
\text { visit." }\end{array}$ & $\begin{array}{l}\text { Step 2. Initial } \\
\text { "weight-prioritized } \\
\text { visits" need to be } \\
\text { scheduled at the } \\
\text { clinic. Follow-up } \\
\text { weight-prioritized } \\
\text { visits can be } \\
\text { scheduled on-line or } \\
\text { by Patient Line. }\end{array}$ & $\begin{array}{l}\text { Step 3. Medical } \\
\text { assistants scrubbing } \\
\text { provider schedules } \\
\text { should note a "weight- } \\
\text { prioritized visit" and } \\
\text { make sure the patient } \\
\text { completes the weight } \\
\text { management } \\
\text { questionnaire prior to } \\
\text { their visit. }\end{array}$ & $\begin{array}{l}\text { Step 4. Provider should } \\
\text { know their resources } \\
\text { for education and } \\
\text { consultative support } \\
\text { for weight } \\
\text { management, then use } \\
\text { the weight } \\
\text { management SmartSet } \\
\text { at the time of the visit. }\end{array}$
\end{tabular}

\section{Staff workflow}

Placards may be placed at the front desk of the clinics alerting patients that they may make a weight-prioritized visit if they would like medical assistance with their weight. Each clinic or provider will determine the number of weight-prioritized visits available. Once a patient schedules a weight-prioritized visit, Epic will request they complete a specified weight management questionnaire upon e-check-in prior to their visit. Medical assistants (MAs) will review provider schedules ahead of

Table 2 Key components of PATHWEIGH and PATHWEIGH implementation strategies

\section{PATHWEIGH is a weight management care path built into Epic. It}

\section{includes:}

- Weight-prioritized visit type: Defines the scope of the visit for the patient and provider.

- Time-efficient documentation and data capture: Patient questionnaire completed prior to the visit captures history around weight gain and barriers to weight loss that can guide the patient-clinician conversation and treatment plan.

- Clinical decision support: SmartSet in Epic suggests weight loss approach(es) based on patient BMI and preferences according to the Obesity Society guidelines [32].

- Tracking: Displays progress with weight loss and weight loss maintenance during patient visits to guide the patient-clinician conversation and evolving treatment plan over time.

- Diagnosis and billing: Tool in Epic automatically adds the diagnosis of overweight (for BMI $25-29.9 \mathrm{~kg} / \mathrm{m}^{2}$ ) or obesity (for BMl>30 kg/m²) and links the most common weight-related co-morbidities (according to the Center for Medicare and Medicaid Services) to ensure adequate provider reimbursement.

\section{PATHWEIGH implementation strategies include:}

- Practice facilitation: Practice facilitators drive implementation efforts by working with practice-selected Practice Champions, engaging leadership in goal setting and monitoring along system and practice goals, and utilizing quality improvement techniques for supporting adoption, implementation, and management of PATHWEIGH.

- Clinician education: Online e-learning module on evidence-based weight management treatment which includes 2 credits of continuing medical education.

- Consultation support: built-in to Epic consultation support for clinicians by request; built-in to Epic availability of UpToDate, a subscriptionbased clinical reference. time to ensure patients complete this questionnaire prior to their visit. At the time of the patient visit, MAs will record the patient's weight and vital signs with no other assistance required. Collectively, the workflow maximizes time for the clinician-patient conversation in the context of the Patient-Centered Medical Home [10].

\section{Clinician workflow}

To optimize efficiency and guide care, health care providers are encouraged to use the PATHWEIGH weight management SmartSet in Epic. A SmartSet is a documentation template used in the ambulatory setting comprised of a group of orders and other elements, such as notes, chief complaints, medications, and levels of service charges, that are commonly used together to document a specific type of visit. This SmartSet addresses the following.

1) Diagnosing overweight or obese patients with a BMI $>25 \mathrm{~kg} / \mathrm{m}^{2}$ or $>30 \mathrm{~kg} / \mathrm{m}^{2}$, respectively, recognizing these as bona fide disease states [6]

2) Linking diagnoses for weight-related comorbidities to facilitate reimbursement

3) Pre-populating information from the patient weight management questionnaire to serve as documentation and to guide the clinician-patient discussion, and flagging weight management strategies based on guidelines from the Obesity Society [32], specifically, the discussion of lifestyle change for patients with $B M I \geq 25 \mathrm{~kg} / \mathrm{m}^{2}$, consideration of medications impacting obesity as well as antiobesity medication at BMI $\geq 30 \mathrm{~kg} / \mathrm{m}^{2}$ (or BMI $\geq 27$ $\mathrm{kg} / \mathrm{m}^{2}$ with weight-related comorbidities) and consideration of bariatric surgery for BMI $\geq 40 \mathrm{~kg} / \mathrm{m}^{2}$ (or BMI $\geq 35 \mathrm{~kg} / \mathrm{m}^{2}$ with weight-related comorbidities)

4) Providing options for orders that include laboratory testing, referrals, or procedures 
Behavioral health professionals work alongside many of the primary care providers and are encouraged adjuncts to facilitate weight loss. Use of the PATHWEIGH tools is entirely optional for clinicians. Use may be deployed by the clinicians in advance of the visit or if they or the patient spontaneously decides to prioritize weight management. The process strongly encourages regular follow-up (usually at 4-6 week intervals and more often initially). A key advantage is that the clinician is the patient's regular provider and will have an ongoing relationship with the patient over time and can safely manage or discontinue medications or other issues for weight-related comorbidities.

\section{Implementation framework and strategies \\ Conceptual framework}

Implementation and evaluation of PATHWEIGH is guided by the Practical, Robust, Implementation and Sustainability Model (PRISM) [33]. RE-AIM (Reach, Effectiveness, Adoption, Implementation, and Maintenance) has often been used as a planning and evaluation framework for capturing important measures in implementation research, but it lacks measures of context and drivers of success [34-37]. PRISM was developed to address the issues of multi-level features for successful program design, predictors of implementation success, factors associated with diffusion, implementation, and maintenance [33]. The key contextual factors for examination include the interaction of system and patient levels with the intervention (includes model components of recipients and intervention), infrastructure elements related to implementation and sustainability (model element of implementation sustainability and infrastructure), and external/environmental factors (model element of external environment) and how they exert an influence on the RE-AIM outcomes.

\section{Implementation strategies}

Implementation strategies are interventions designed to improve the effectiveness of PATHWEIGH by promoting its adoption and use [38, 39]. These strategies were based on our development work, where key stakeholders identified the following three main areas of focus for PATHWEIGH implementation: (1) training using an elearning module including continuing medical education (CME) credit for providers on how to counsel patients on weight loss and how to prescribe evidence-based weight loss treatments, (2) consultation support by experts in weight management built into Epic, and (3) assistance from practice facilitators available to each clinic to identify clinic champions and to assure that PATHWEIGH gets incorporated into workflows, becomes known to patients, and is monitored using quality improvement (QI) principles. All implementation strategies are deployed to clinics when they are randomly assigned to begin using PATHWEIGH. These are common resources available in other health systems to facilitate eventual dissemination.

\section{Evaluation}

Aim 1: Compare the effectiveness of PATHWEIGH versus standard of care $(\mathrm{SOC})$ on weight loss and weight loss maintenance.

\section{Outcomes and Measures}

The primary outcome is patient weight between baseline and 6 months in kilograms $(\mathrm{kg})$ as measured by a standard scale. The secondary outcome of weight loss maintenance will be defined at 12 and 18 months as a binary outcome of weight increase $\leq 10 \%$ from the patient's 6 month weight. Weight outcomes will be extracted from the EMR. The primary independent variable will be the intervention status, which will be determined based on visit date and practice sequence. Additional covariate data will be collected from the EMR on potential confounders at the level of patient, provider, and practice. Patient-level data will include demographic information, health metrics, behaviors and goals, and treatment strategy employed. Provider-level data will include demographic information, years practicing, and percent time practicing. Clinic-level data will include site type/discipline, location, and size. Outcomes, measures, and timing of data collection are described in Table 3.

\section{Analysis}

A generalized linear mixed modeling strategy will be employed to account for multiple levels of correlation (i.e., between patients within practices and between measurements on a single patient over time) [44]. This approach allows for the most efficient use of data if any follow-up measurements are missing for some subjects. We will follow an intention-to-treat approach for our primary analysis, i.e., patients will be analyzed according to their clinic's intervention condition (PATHWEIGH vs. SOC) at the time of the patient's index visit. An index visit will be defined as the patient's first weightprioritized visit in an intervention condition. Follow-up visits at which weight is recorded will be associated with the intervention status of the patient's preceding index visit.

For the primary analysis, although the parameter of interest is weight change at 6 months, weight collected from baseline to approximately 9 months post-baseline for the individual patient will be included in the primary analysis because patients may not have visits with recorded weight within the 6-month time frame. The mixed model will include random intercepts for patient, provider, and clinic, along with fixed effects for time and intervention condition. The time effects are two-fold: (i) 
Table 3 Summary of outcomes, measures, data sources, and timing of collection for PATHWEIGH study

\begin{tabular}{llll}
\hline Construct & Metric/measure & Method of collection & Timing \\
\hline $\begin{array}{c}\text { Patient-specific outcomes } \\
\text { Weight-related outcomes }\end{array}$ & $\begin{array}{l}\text { Weight }(\mathrm{kg}) \text {, height }(\mathrm{cm}), \text { weight loss } \\
\text { maintenance (weight increase } \leq 10 \%)\end{array}$ & EMR & Collected during routine care \\
Clinical and laboratory values & $\begin{array}{l}\text { Thyroid-stimulating hormone }(\mathrm{TSH}), \text { lipid } \\
\text { panel, liver function tests, A1c, blood } \\
\text { pressure }\end{array}$ & EMR & Collected during routine care \\
Patient characteristics & $\begin{array}{l}\text { Demographics (age, gender, race/ } \\
\text { ethnicity, insurance, comorbidities, } \\
\text { medications) }\end{array}$ & EMR & Collected during routine care
\end{tabular}

\section{Clinician and clinic staff-specific outcomes}

Clinic characteristics
Practice culture
Implementation climate
History of practice improvement
Self-efficacy and satisfaction

\section{RE-AIM Outcomes}

Reach - The absolute number, proportion, and representativeness of individuals who participate

Effectiveness - The impact of an intervention on important patient outcomes

Adoption - The absolute number, proportion, and representativeness of settings and providers who initiate the intervention

Use of PATHWEIGH by any provider in a clinic (setting level) and each provider (provider level)

Factors influencing adoption/ implementation

Implementation - The intervention agents' fidelity to the various elements of the intervention's protocol including consistency of delivery as intended and the time and cost of the intervention

Fidelity to PATHWEIGH functional core components and $\mathrm{SOC}$ in control clinics; use of implementation strategies; Clinician use of PATHWEIGH for $>50 \%$
\# and characteristics of clinicians and patients empaneled to the clinic

Practice culture scale $[40,41]$

Implementation climate scale $[42,43]$

\# and type of practice improvement in the past year

One item assessment of perceived confidence in ability to provide weight management, one item assessment of perceived satisfaction

\#/characteristics of patients in

EMR

PATHWEIGH out of weight-prioritized and eligible per clinic

Barriers, facilitators to participation and outcomes

Interviews with selected patients

Survey to practice leadership

Survey to providers and staff

Survey to providers and staff

Survey to providers and staff

Survey to providers and staff

Evaluated using weight-related outcomes described above. weight-prioritized visits; e-learning module completion per clinician; use of consultation; participation in PF trainings

Acceptability of PATHWEIGH; adaptations

Barriers, facilitators to implementation

Cost and resources for implementation
Survey to practice leadership (practice characteristics), EMR (provider use of PATHWEIGH)

Interviews with selected providers and staff

EMR

Survey to all providers, some staff, and selected patients

Interviews with selected providers, clinic leaders, and staff

Cost/resources interviews with practice managers and selected provider/staff by research assistant
Baseline

Baseline and 1-year postintervention

Baseline and 1-year postintervention

Baseline and 1-year postintervention

Baseline and 1-year postintervention

Baseline (patient characteristics) and at each 1-year step (patient participation)

6-month post-intervention start for the patient

Baseline and at each 1-year step

Baseline and 1-year postintervention start for each sequence

Ongoing collection; review at 6month post-intervention start and study end; completion of implementation strategies

6- month post-intervention start for that cohort

1-year post-intervention start for each cohort

Control condition and 1-year post-intervention start for each cohort 
Table 3 Summary of outcomes, measures, data sources, and timing of collection for PATHWEIGH study (Continued)

\begin{tabular}{llll}
\hline Construct & Metric/measure & Method of collection & Timing \\
\hline $\begin{array}{l}\text { Maintenance - The extent to which } \\
\text { the intervention becomes part of } \\
\text { practice }\end{array}$ & $\begin{array}{l}\text { Plans to continue/adapt PATHWEIGH past } \\
\text { study }\end{array}$ & $\begin{array}{l}\text { Survey and interviews with } \\
\text { practice managers and } \\
\text { selected providers and staff }\end{array}$ & $\begin{array}{l}\text { 1-year post-intervention start for } \\
\text { eachort; and study end }\end{array}$ \\
\hline
\end{tabular}

Abbreviations: EMR electronic medical record, PF practice facilitator, SOC standard of care

calendar time, divided into four 1-year periods and included as a categorical variable to flexibly model its effect on the outcome and account for temporal trends, and (ii) individual patient time, included as a continuous variable (i.e., time from baseline) to accommodate varying visit times determined by each patient's visit schedule. The intervention condition variable will be a binary indicator that is equal to zero if the patient's index visit occurs at a clinic when it is in the control condition and becomes equal to 1 when the patient has an index visit at a clinic after it has crossed into the intervention condition. Patient-, provider-, and practice-level covariates will be included in models if deemed to be potential confounders in univariate models. An additional fixed effect for the interaction between patient time and intervention condition will be used to estimate the parameters of primary interest, i.e., the difference in change in outcomes over time between the intervention and control conditions. The primary analysis will test the null hypothesis that the interaction effect coefficient is equal to zero to determine whether there is a significant difference in weight change between the PATHWEIGH and SOC conditions. Using the model, we will report the mean weight change in the control and intervention groups and their difference at 6 months, with corresponding 95\% confidence intervals and $p$ values.

To examine the secondary binary outcomes of weight maintenance (defined as $<10 \%$ weight gain at 12 and 18 months), we will use generalized linear mixed models. Since this analysis will involve a single outcome per patient, the model will only include a random intercept for provider and clinic. The test of intervention effectiveness will be captured by the fixed effect term for the intervention condition.

The primary analysis is expected to provide a conservative estimate of the treatment effect, and we propose the following secondary analyses to assess the treatment effect in alternative cohorts. We will perform a perprotocol analysis that uses the described models to compare those that received PATHWEIGH to those with a SOC weight-prioritized visit. This analysis will provide a better estimate of the efficacy of the intervention by including only those in the PATHWEIGH condition that were considered to satisfy the criteria for receiving the intervention as assessed by the provider's use of at least one of a weight prioritized visit "type", the patient weight management questionnaire, and/or the weight management SmartSet. Additionally, we will perform an analysis using all those that are eligible for PATHWEIGH based on the criteria of age and BMI, regardless of whether they had a weight-prioritized visit. This analysis will allow us to account for the potential selection bias induced by the implementation of PATHWEIGH potentially increasing the number of weight-prioritized visits and thus increasing the enrollment of patients in the intervention period that differ systematically from those in the control period.

\section{Aim 2: Identify patient, provider, and clinic-level predictors that are associated with weight loss and weight loss maintenance Outcomes and measures}

Patient-level predictors will be captured from the EMR for each visit. Potential patient-level moderators will include age, sex, race/ethnicity, insurance status, baseline BMI, baseline use of medication that may contribute to weight gain, and score $>9$ on Patient Health Questionnaire-8 or -9 (PHQ8, PHQ9), or Generalized Anxiety Disorder-7 (GAD7). Patient-level mediators will include treatment prescribed, in particular prescription of anti-obesity medication and performance of an endoscopic or surgical procedure with bariatrics. Providerlevel predictors that can be obtained from EMR include provider age, sex, years in practice, and percent time performing clinical duties. Practice-level predictors include patient and provider number and demographics for each, as well as payor mix.

\section{Analysis}

Moderation will be evaluated by including an interaction term between the treatment variable and possible moderating variables in the outcome mixed effects regression models used in Aim 1. A significant interaction effect will conclude that the considered variable moderates the effect of PATHWEIGH on the outcome. Mediation analysis will be conducted using the multi-level modeling framework to account for clustering within clinics and providers $[45,46]$. Clinic- and provider-level mediators will be restricted to those that can be collected from EMR in both the control and intervention phases. Specifically, we will fit two models: (1) a mediator model to describe the effect of the treatment on the mediator, and (2) an outcomes model to describe the effect of the mediator on the outcome, adjusting for the treatment. 
Additionally, to be considered a mediator, change in the mediator variable must occur prior to or during the period of patient-level change. Both models will be adjusted for relevant patient-, provider-, and clinic-level variables. The outcomes model will additionally adjust for a time effect to account for temporal effects in the stepped wedge design. These models will be adjusted to include the appropriate random effects and variables based on the level of the considered mediator variable (i.e., patient, provider, clinic). The mediation effect will be summarized as the product of the treatment coefficient from the mediator model and the mediator coefficient from the outcomes model. We will test the null hypothesis that this product is equal to zero, and a statistically significant result will indicate that there is strong evidence that the effect of the treatment on the outcome is mediated by the considered variable. If necessary, we will explore more complex multi-level structural equation models [47].

\section{Aim 3: Describe factors associated with practice adoption, implementation, and maintenance of PATHWEIGH. Outcomes and Measures}

Practice member surveys A baseline (pre-intervention) survey will be given to all practice members to assess personal characteristics (PRISM element of recipients) and practice culture, implementation climate, and perception of system priority for weight management (PRISM elements of implementation and sustainability infrastructure, and intervention). Clinicians will also be assessed on current weight management practices and their self-efficacy and satisfaction with obesity treatment (PRISM elements of recipients and intervention). These surveys will be repeated at 1 year after PATHWEIGH implementation for comparison pre-post intervention.

Provider, staff, and patient interviews A purposeful selection of providers and staff, estimated at three to six total per practice, will be interviewed to assess RE-AIM outcomes. Practice staff and clinician interviews will focus on factors influencing adoption of PATHWEIGH and use of the PATHWEIGH implementation strategies, as well as influences on appropriate use of PATHWEIGH. Interviews will seek to understand the context of the delivery as well as the mindsets and belief systems driving thoughts and actions about what happens. External influences such as financial demands and staff turnover will be explored as potential sources of implementation difficulty (PRISM elements of recipients, intervention, implementation and sustainability infrastructure, external environment). Patient interviews (estimated at 40 total per year for each of the three cohorts) will explore their perspectives and experience with the intervention.

Other data collection Observations will be conducted by research assistants at 3-6 months post-implementation. Research assistants will shadow selected patient visits to determine fidelity and quality of patient-provider communication on weight. A fidelity checklist and extensive field notes will be used. General practice observations will also be conducted to illuminate how the implementation works in practice and where there may be leakage, slippage, or gaps occurring in care processes in the intervention. Use of PATHWEIGH will also be extracted from Epic during the scheduled data query periods to determine clinicians' use of PATHWEIGH tools as a measure of adoption. Additionally, research assistants will conduct key informant interviews with selected staff in practices to assess the cost and resources needed to implement PATHWEIGH and the associated implementation strategies. To assess financial sustainability, clinical billing data will be extracted from Health Data Compass to assess reimbursement for the weight-prioritized visits using standardized Medicare reimbursement rates.

\section{Analysis}

Quantitative analysis of the reach, adoption, implementation, and maintenance outcomes in Table 3 will be conducted using survey responses and fidelity checklists. Data will be analyzed descriptively (e.g., means, proportions) and estimated pre- and post-intervention. Inferential comparisons regarding association of the intervention with outcomes will be based on paired $t$ tests (continuous variables), McNemar's chi-square tests (categorical variables), or generalized mixed modeling to adjust for potential confounders.

Qualitative methods, including interviews and observations, will be used to determine contributions to the adoption, implementation, and maintenance outcomes and PRISM contextual factors affecting these outcomes. Interviews will be audio-recorded, transcribed, cleaned, and entered into the qualitative software program, ATLAS.ti. We will use a grounded theory hermeneutic editing approach [48] to identify issues regarding intervention implementation from the perspectives of providers, staff members, leaders, and patients. Codes will be identified and created based on emergent elements in the data and will be used to tag relevant text from the transcripts. Quotation reports, which list all the associated quotations verbatim, will be generated and then organized by practice. The qualitative core group will hold ongoing meetings to read through all the quotations for these codes and categorize text that exemplifies the identified constructs. After initial thematic groupings, member-checking will be completed with key practice 
members. Additionally, we will use a matrix approach to determine patterns of emergent concepts across PRISM contextual factors and across practices.

Using a mixed methods concurrent parallel design, each element of data collection will occur separately [49]. Specifically, quantitative data will be collected and analyzed, and the qualitative data will be collected and analyzed. Then the two sets of data will be analyzed and interpreted together. Specific methods will include both a matrix approach and a data transformation approach to mixing of the data. Joint display tables describing the interplay of the quantitative and qualitative results will be created [50]. To achieve our aims, we will use the analysis technique of qualitative comparative analysis (QCA) [51]. QCA applies set theory to identify necessary and one or more sufficient sets of conditions to produce favorable outcomes, in this case, to determine what sorts of practice conditions and structures were needed to effectively implement PATHWEIGH [51, 52]. The fuzzyset variant (fsQCA) allows simultaneous use of qualitative and quantitative data. It is newly applied to practice transformation [53], and we are using it in other work [54]. We will use this analysis to merge information from all sources and evaluate the contribution of specific PRISM factors on the outcome of intervention implementation and our practice break-even measure from the cost analysis. We will use the process detailed by Rihoux and Ragin and the fsQCA software [51].

\section{Return on investment}

A formal return on investment (ROI) analysis will estimate the cost associated with adopting, implementing, and maintaining the intervention in practices and compare this to the difference in reimbursements before and after implementation in each practice. We will adapt a time-driven activity-based costing questionnaire that we have used in several previous studies $[55,56]$ for the estimation of the cost of staff time used in delivering the SOC and PATHWEIGH visits (including time of PATHWEIGH consulting obesity certified providers), practice facilitation support, and time clinic staff participate in training. In addition, we will measure the cost of materials provided to patients, hiring costs (if any), and other administrative costs incurred for all aspects of PATHWEIGH. To generalize to other settings, we will use industry average compensation costs for providers and staff to value time-driven costs as well as health system average compensation costs for the ROI analysis. Reimbursement data from the health system billing system will be collected for both the control and intervention period. We will collect clinic-level revenue as well as the Medicare allowed amounts for weight-prioritized visits. In addition, for the system-level ROI, we will obtain total system revenue for those patients that have a weight-prioritized visit in each calendar year. A practice perspective ROI will be calculated comparing the PATHWEIGH implementation cost to the difference in reimbursement for weight-prioritized visits during PATHWEIGH implementation and control periods. A system perspective ROI will compare the PATHWEIGH implementation cost to the difference in annualized revenue for patients with a weight-prioritized visit under the PATHWEIGH and control condition.

\section{Dissemination}

PATHWEIGH was designed for dissemination [57]. The University of Colorado has trademarked PATHWEIGH so deployment through the large national network of Epic users is possible. This was not intended to generate revenue, but rather so data use agreements can be drafted that ensure data flow back to the study team so iterations can be made that improve its usefulness for all users. The e-learning module and practice facilitator training materials will be made available. Our team has experience with developing and disseminating implementation products [58]. Also, we will share results at scholarly meetings and in peer-reviewed journals. Deidentified data sets used in analyses will be made available upon request.

\section{Trial status}

This manuscript describes the study protocol (version 1 , July 6,2021 ) reviewed and funded by the National Institutes of Health (NIH) in September 2020. Enrollment to the study began in March 2021 and at the time of submission the research team is implementing PATHWEIGH in the first sequence of clinics. Recruitment and data collection will be completed in September 2025 .

\section{Discussion}

The goal of PATHWEIGH is to make weight management effective for patients and feasible and satisfying for medical providers and their teams in primary care. By utilizing the power of the electronic medical record to create efficiencies and drive evidence-based care, this goal may be achievable. This study will contribute to the field by testing if this approach accomplishes both effectiveness for patients' health improvement and is adopted by typical clinical practices. It will add important findings about how the implementation strategies in combination contribute to both implementation and effectiveness outcomes. The use of a stepped wedge cluster randomized trial using covariate constrained randomization within the context of an effectivenessimplementation hybrid design is innovative and should advance methodological findings for the field. Although this study should provide findings that will be applicable 
to many settings and will be examined in a large number of diverse practices, this study will only be implemented in a single health system in one state. Therefore, the results may not translate to other circumstances and other settings, and contextual adaptation may be necessary. However, if successful, results may be disseminated through Epic, the most widely available electronic medical record system in the U.S. According to the Epic User Web, such a system is not yet available nor has been tested. Thus, this study could make a significant impact on weight and the resultant comorbidities experienced by Americans.

\begin{abstract}
Abbreviations
BMI: Body mass index; CDC: Centers for Disease Control and Prevention; CME: Continuing medical education; EMR: Electronic medical record; FM: Family Medicine; fsQCA: Fuzzy-set Qualitative comparative analysis; GIM: General Internal Medicine; HPI: History of present illness; IBT: Intensive behavioral therapy; ICC: Intraclass correlation coefficient; MA: Medical assistant; NIH: National Institutes of Health; PRISM: Practical, Robust, Implementation and Sustainability Model; QCA: Qualitative comparative analysis; Ql: Quality improvement; RE-AIM: Reach, Effectiveness, Adoption, Implementation, Maintenance; ROI: Return on investment; SD: Standard deviation; SOC: Standard of care
\end{abstract}

\section{Supplementary Information}

The online version contains supplementary material available at https://doi. org/10.1186/s13063-021-05954-7.

Additional file 1: Covariate Constrained Randomization. Fig. S1 Standard of Care (Obesity brief HPI) initial and follow-up questionnaires.

\section{Acknowledgements}

The study team acknowledges the indispensable assistance and support of the Health Data Compass Data Warehouse project (healthdatacompass.org) and the leadership team at the University of Colorado Health, without which this project would not be possible.

\section{Authors' contributions}

$L M D, R M G, J H, L P$, and PCS contributed to conceptualizing the study and writing the original study proposal on which this manuscript is based. LMD, RMG, JH, LP, PCS, KS, and EW contributed to writing, reviewing, and editing this manuscript. All authors have read and approved the manuscript.

\section{Funding}

Research reported in this manuscript was funded through a National Institutes of Health (NIH) Award (1R18DK127003).

\section{Availability of data and materials}

Not applicable.

\section{Declarations}

\section{Ethics approval and consent to participate}

Ethics approval was granted by the Colorado Multiple Institutional Review Board (COMIRB; 20-1954). A written informed consent process was utilized for only specified, individually contacted patients and practice members. Other data were de-identified and therefore informed consent was not utilized.

\section{Consent for publication}

Not applicable.

\section{Competing interests}

LP has received personal fees for consulting and/or speaking from Novo Nordisk, Sanofi, Elli Lilly, Boehringer Ingelheim, Astra Zeneca, Medscape,
WebMD, and UpToDate. LMD, RMG, JH, PCS, KS, and EW have no competing interests.

\section{Author details}

${ }^{1}$ Colorado School of Public Health, 13001 East 17th Place, Aurora, CO 80045, USA. ${ }^{2}$ University of Colorado School of Medicine, 13199 E Montview Blvd Ste 300, Aurora, CO 80045, USA.

Received: 21 August 2021 Accepted: 20 December 2021

Published online: 10 January 2022

\section{References}

1. Adult Obesity Facts. https://www.cdc.gov/obesity/data/adult.html. Accessed 22 March 2021.

2. Obesity. https://www.who.int/topics/obesity/en/. Accessed 22 March 2021.

3. Stefan N, Birkenfeld AL, Schulze MB, Ludwig DS. Obesity and impaired metabolic health in patients with COVID-19. Nat Rev Endocrinol. 2020;16(7): 341-2. https://doi.org/10.1038/s41574-020-0364-6.

4. Economic impact of excess weight now exceeds \$17 T 2018. https://www. milkeninstitute.org/articles/economic-impact-excess-weight-now-exceeds-1 7-trillion-new-milken-institute-report-reveals. Accessed 23 July 2019.

5. Crawford AG, Cote C, Couto J, Daskiran M, Gunnarsson C, Haas K, et al. Prevalence of obesity, type II diabetes mellitus, hyperlipidemia, and hypertension in the United States: findings from the GE Centricity Electronic Medical Record database. Popul Health Manag. 2010;13(3):151-61. https:// doi.org/10.1089/pop.2009.0039.

6. Garvey WT, Mechanick JI, Brett EM, Garber AJ, Hurley DL, Jastreboff AM, et al. American Association of Clinical Endocrinologists and American College of Endocrinology Comprehensive Clinical Practice Guidelines for Medical Care of Patients with Obesity. Endocr Pract. 2016;22(Suppl 3):1-203. https://doi.org/10.4158/EP161365.GL.

7. Kaplan LM, Golden A, Jinnett K, Kolotkin RL, Kyle TK, Look M, et al. Perceptions of Barriers to Effective Obesity Care: Results from the National ACTION Study. Obesity (Silver Spring). 2018;26(1):61-9. https://doi.org/10.1 002/oby.22054.

8. Kushner RF. Barriers to providing nutrition counseling by physicians: a survey of primary care practitioners. Prev Med. 1995;24(6):546-52. https:// doi.org/10.1006/pmed.1995.1087.

9. Wadden TA, Volger S, Tsai AG, Sarwer DB, Berkowitz RI, Diewald LK, et al. Managing obesity in primary care practice: an overview with perspective from the POWER-UP study. Int J Obes (Lond). 2013;37(Suppl 1):S3-11. https://doi.org/10.1038/ijo.2013.90.

10. Rosenberg CN, Peele P, Keyser D, McAnallen S, Holder D. Results from a patient-centered medical home pilot at UPMC Health Plan hold lessons for broader adoption of the model. Health Aff (Millwood). 2012;31(11):2423-31. https://doi.org/10.1377/hlthaff.2011.1002.

11. Leblanc ES, O'Connor E, Whitlock EP, Patnode CD, Kapka T. Effectiveness of primary care-relevant treatments for obesity in adults: a systematic evidence review for the U.S. Preventive Services Task Force. Ann Intern Med. 2011; 155(7):434-47. https://doi.org/10.7326/0003-4819-155-7-201110040-00006.

12. Bodenheimer T, Young DM, MacGregor K, Holtrop JS. Practice-based research in primary care: facilitator of, or barrier to, practice improvement? Ann Fam Med. 2005;3(Suppl 2):S28-32. https://doi.org/10.1370/afm.341.

13. Woolf SH, Glasgow RE, Krist A, Bartz C, Flocke SA, Holtrop JS, et al. Putting it together: finding success in behavior change through integration of services. Ann Fam Med. 2005;3(Suppl 2):S20-7. https://doi. org/10.1370/afm.367.

14. Sherson EA, Yakes Jimenez E, Katalanos N. A review of the use of the 5 A's model for weight loss counselling: differences between physician practice and patient demand. Fam Pract. 2014;31(4):389-98. https://doi.org/10.1093/ fampra/cmu020

15. Yoong SL, Carey M, Sanson-Fisher R, Grady A. A systematic review of behavioural weight-loss interventions involving primary-care physicians in overweight and obese primary-care patients (1999-2011). Public Health Nutr. 2013;16(11):2083-99. https://doi.org/10.1017/S1368980012004375.

16. Bohula EA, Scirica BM, Inzucchi SE, McGuire DK, Keech AC, Smith SR, et al. Effect of lorcaserin on prevention and remission of type 2 diabetes in overweight and obese patients (CAMELLIA-TIMI 61): a randomised, placebocontrolled trial. Lancet. 2018;392(10161):2269-79. https://doi.org/10.1016/ S0140-6736(18)32328-6. 
17. Bohula EA, Wiviott SD, McGuire DK, Inzucchi SE, Kuder J, Im K, et al. Cardiovascular Safety of Lorcaserin in Overweight or Obese Patients. N Engl J Med. 2018;379(12):1107-17. https://doi.org/10.1056/NEJMoa1808721.

18. Garber AJ. Anti-Obesity Pharmacotherapy and the Potential for Preventing Progression from Prediabetes to Type 2 Diabetes. Endocr Pract. 2015;21(6): 634-44. https://doi.org/10.4158/EP14460.RA.

19. Svanstrom H, Ueda P, Melbye M, Eliasson B, Svensson AM, Franzen S, et al Use of liraglutide and risk of major cardiovascular events: a register-based cohort study in Denmark and Sweden. Lancet Diabetes Endocrinol. 2019; 7(2):106-14. https://doi.org/10.1016/S2213-8587(18)30320-6.

20. Carlsson LM, Sjoholm K, Karlsson C, Jacobson P, Andersson-Assarsson JC, Svensson PA, et al. Long-term incidence of microvascular disease after bariatric surgery or usual care in patients with obesity, stratified by baseline glycaemic status: a post-hoc analysis of participants from the Swedish Obese Subjects study. Lancet Diabetes Endocrinol. 2017;5(4):271-9. https:// doi.org/10.1016/S2213-8587(17)30061-X.

21. Fisher DP, Johnson E, Haneuse S, Arterburn D, Coleman KJ, O'Connor PJ, et al. Association Between Bariatric Surgery and Macrovascular Disease Outcomes in Patients With Type 2 Diabetes and Severe Obesity. JAMA. 2018;320(15):1570-82. https://doi.org/10.1001/jama.2018.14619.

22. Inge TH, Courcoulas AP, Jenkins TM, Michalsky MP, Helmrath MA, Brandt ML, et al. Weight Loss and Health Status 3 Years after Bariatric Surgery in Adolescents. N Engl J Med. 2016;374(2):113-23. https://doi.org/10.1056/ NEJMoa1506699.

23. Sjostrom L, Narbro K, Sjostrom CD, Karason K, Larsson B, Wedel H, et al. Effects of bariatric surgery on mortality in Swedish obese subjects. N Engl J Med. 2007;357(8):741-52. https://doi.org/10.1056/NEJMoa066254.

24. Intensive behavioral therapy (IBT) for obesity. https://www.cms.gov/Medica re/Prevention/PrevntionGenInfo/medicare-preventive-services/MPSQuickReferenceChart-1.htm|\#OBESITY_IBT. Accessed 5 Feb 2020.

25. Curran GM, Bauer M, Mittman B, Pyne JM, Stetler C. Effectivenessimplementation hybrid designs: combining elements of clinical effectiveness and implementation research to enhance public health impact. Med Care. 2012;50(3):217-26. https://doi.org/10.1097/MLR.0b013e31 82408812.

26. Hussey MA, Hughes JP. Design and analysis of stepped wedge cluster randomized trials. Contemp Clin Trials. 2007;28(2):182-91. https://doi.org/1 0.1016/j.cct.2006.05.007.

27. Chan AW, Tetzlaff JM, Gotzsche PC, Altman DG, Mann H, Berlin JA, et al. SPIRIT 2013 explanation and elaboration: guidance for protocols of clinical trials. BMJ. 2013;346(jan08 15):e7586. https://doi.org/10.1136/bmj.e7586.

28. Hemming K, Taljaard M, McKenzie JE, Hooper R, Copas A, Thompson JA, et al. Reporting of stepped wedge cluster randomised trials: extension of the CONSORT 2010 statement with explanation and elaboration. BMJ. 2018; 363:k1614. https://doi.org/10.1136/bmj.k1614.

29. Perreault L, Hockett C, Holmstrom H, Tolle L, Kramer E. PATHWEIGH Tool for Chronic Weight Management Built into EPIC Electronic Medical Record: Methods, Pilot Results and Future Directions. J Obes Chronic Dis. 2020;4(1): 42-8. https://doi.org/10.17756/jocd.2020-036.

30. Chaussee EL, Dickinson LM, Fairclough DL. Evaluation of a covariateconstrained randomization procedure in stepped wedge cluster randomized trials. Contemp Clin Trials. 2021;105:106409. https://doi.org/10.1 016/j.cct.2021.106409.

31. Glasgow RE, Huebschmann AG, Brownson RC. Expanding the CONSORT figure: increasing transparency in reporting on external validity. Am J Prev Med. 2018;55(3):422-30. https://doi.org/10.1016/j.amepre.2018.04.044.

32. Jensen MD, Ryan DH, Apovian CM, Ard JD, Comuzzie AG, Donato KA, et al. 2013 AHA/ACC/TOS guideline for the management of overweight and obesity in adults: a report of the American College of Cardiology/American Heart Association Task Force on Practice Guidelines and The Obesity Society. J Am Coll Cardiol. 2014;63(25 Part B):2985-3023.

33. Feldstein AC, Glasgow RE. A practical, robust implementation and sustainability model (PRISM) for integrating research findings into practice. Jt Comm J Qual Patient Saf. 2008;34(4):228-43. https://doi.org/10.1016/S1 553-7250(08)34030-6.

34. Gaglio B, Shoup JA, Glasgow RE. The RE-AIM framework: a systematic review of use over time. Am J Public Health. 2013;103(6):e38-46. https://doi.org/1 0.2105/AJPH.2013.301299.

35. Glasgow RE, Vogt TM, Boles SM. Evaluating the public health impact of health promotion interventions: the RE-AIM framework. Am J Public Health. 1999;89(9):1322-7. https://doi.org/10.2105/AJPH.89.9.1322.
36. Kessler RS, Purcell EP, Glasgow RE, Klesges LM, Benkeser RM, Peek CJ. What does it mean to "employ" the RE-AIM model? Eval Health Prof. 2013;36(1): 44-66. https://doi.org/10.1177/0163278712446066.

37. Shoup JA, Gaglio B, Varda D, Glasgow RE. Network analysis of RE-AIM framework: chronology of the field and the connectivity of its contributors. Transl Behav Med. 2015;5(2):216-32. https://doi.org/10.1007/s13142-014-03 00-1.

38. Kirchner JEWT, Powell BJ, Smith JL, Proctor EK. Implementation strategies. In: Brownson RC, Colditz GA, Proctor EK, editors. Dissemination and Implementation Research in Health: Translating Science to Practice. 2nd ed. New York: Oxford University Press; 2018.

39. Powell BJ, Waltz TJ, Chinman MJ, Damschroder LJ, Smith JL, Matthieu MM, et al. A refined compilation of implementation strategies: results from the Expert Recommendations for Implementing Change (ERIC) project. Implement Sci. 2015;10(1):21. https://doi.org/10.1186/s13012-015-0209-1.

40. Dickinson WP, Dickinson LM, Nutting PA, Emsermann CB, Tutt B, Crabtree $B F$, et al. Practice facilitation to improve diabetes care in primary care: a report from the EPIC randomized clinical trial. Ann Fam Med. 2014;12(1):816. https://doi.org/10.1370/afm.1591.

41. Dickinson LM, Dickinson WP, Nutting PA, Fisher L, Harbrecht M, Crabtree BF, et al. Practice context affects efforts to improve diabetes care for primary care patients: a pragmatic cluster randomized trial. J Gen Intern Med. 2015; 30(4):476-82. https://doi.org/10.1007/s11606-014-3131-3.

42. Ehrhart MG, Aarons GA, Farahnak LR. Assessing the organizational context for EBP implementation: the development and validity testing of the Implementation Climate Scale (ICS). Implement Sci. 2014;9(1):157. https:// doi.org/10.1186/s13012-014-0157-1.

43. Jacobs SR, Weiner BJ, Bunger AC. Context matters: measuring implementation climate among individuals and groups. Implement Sci. 2014;9(1):46. https://doi.org/10.1186/1748-5908-9-46.

44. Fitzmaurice GM, Laird NM, Ware JH. Applied longitudinal analysis. 2nd ed. Hoboken, N.J.: Wiley; 2011. https://doi.org/10.1002/9781119513469.

45. Bauer DJ, Preacher KJ, Gil KM. Conceptualizing and testing random indirect effects and moderated mediation in multilevel models: new procedures and recommendations. Psychol Methods. 2006;11(2):142-63. https://doi. org/10.1037/1082-989X.11.2.142.

46. Kraemer HC, Wilson GT, Fairburn CG, Agras WS. Mediators and moderators of treatment effects in randomized clinical trials. Arch Gen Psychiatry. 2002; 59(10):877-83. https://doi.org/10.1001/archpsyc.59.10.877.

47. Preacher KJ, Zyphur MJ, Zhang Z. A general multilevel SEM framework for assessing multilevel mediation. Psychol Methods. 2010;15(3):209-33. https:// doi.org/10.1037/a0020141.

48. Addison RB. A grounded hermeneutic editing approach. In: Crabtree BF, Miller WL, editors. Doing Qualitative Research. 2nd ed. Thousand Oaks, CA Sage Publications, Inc; 1999.

49. Creswell JW, Clark VLP. Choosing a mixed methods design. In: Cresswell JW, Clark VLP, editors. Designing and conducting mixed methods research. 2nd ed. Thousand Oaks, CA: Sage Publications; 2011.

50. Guetterman TC, Fetters MD, Creswell JW. Integrating Quantitative and Qualitative Results in Health Science Mixed Methods Research Through Joint Displays. Ann Fam Med. 2015;13(6):554-61. https://doi.org/10.1370/a fm. 1865.

51. Rihoux B, Ragin CC. Configurational comparative methods: qualiative comparative analysis (QCA) and related techniques, vol. 51. Thousand Oaks, CA: Sage Publications, Inc; 2009. https://doi.org/10.4135/9781452226569.

52. Ragin CC. Studying cases as configurations. In: Fuzzy-set social science. 1st ed. Chicago, IL: University of Chicago Press; 2000.

53. Marcus Thygeson N, Solberg LI, Asche SE, Fontaine P, Gregory Pawlson L, Scholle SH. Using fuzzy set qualitative comparative analysis (fs/QCA) to explore the relationship between medical "homeness" and quality. Health Serv Res. 2012;47(1 Pt 1):22-45. https://doi.org/10.1111/j.1475-6773.2011.01303.x.

54. Holtrop JS, Hall TL, Rubinson C, Dickinson LM, Glasgow RE. What Makes for Successful Registry Implementation: A Qualitative Comparative Analysis. J Am Board Fam Med. 2017;30(5):657-65. https://doi.org/10.3122/jabfm.2017. 05.170096.

55. Kaplan RS, Anderson SR. Time-driven activity-based costing. Harv Bus Rev. 2004;82(11):131-8

56. Kaplan RS, Witkowski M, Abbott M, Guzman AB, Higgins LD, Meara JG, et al. Using time-driven activity-based costing to identify value improvement opportunities in healthcare. J Healthc Manag. 2014;59(6):399-412. https:// doi.org/10.1097/00115514-201411000-00005 
57. Brownson RC, Jacobs JA, Tabak RG, Hoehner CM, Stamatakis KA. Designing for dissemination among public health researchers: findings from a national survey in the United States. Am J Public Health. 2013;103(9):1693-9. https:// doi.org/10.2105/AJPH.2012.301165.

58. Kwan BM, Fernald D, Ferrarone P, Loskutova N, Summers Holtrop J, Staton EW, et al. Implementation and Evaluation of a Laboratory Safety Process Improvement Toolkit. J Am Board Fam Med. 2019;32(2):136-45. https://doi org/10.3122/jabfm.2019.02.180109.

\section{Publisher's Note}

Springer Nature remains neutral with regard to jurisdictional claims in published maps and institutional affiliations.

Ready to submit your research? Choose BMC and benefit from:

- fast, convenient online submission

- thorough peer review by experienced researchers in your field

- rapid publication on acceptance

- support for research data, including large and complex data types

- gold Open Access which fosters wider collaboration and increased citations

- maximum visibility for your research: over $100 \mathrm{M}$ website views per year

At $\mathrm{BMC}$, research is always in progress.

Learn more biomedcentral.com/submissions 